\title{
Biliary Ascariasis Pancreatitis
}

\author{
Rosnelifaizur Ramely, Mohd Nizam Mohd Hashim, Wan Zainira Wan Zin, \\ Ikhwan Sani Mohamad, Syed Hassan Syed Ab Aziz, \\ Department of Surgery, School of Medical Sciences, Universiti Sains Malaysia, Health Campus, Kelantan,
} Malaysia.

\section{Introduction}

Ascariasis is caused by the nematode, Ascaris lumbricoides, commonly found in the tropics. These parasites are transmitted via the faecal oral route. Their eggs hatch in the small intestines and the larvae migrate through the gut wall into the bloodstream and to the alveoli. They subsequently move up the respiratory tract to the trachea and are swallowed. The larvae mature in the small intestines, deriving nutrients from ingested food. Most infections are asymptomatic. Common manifestations include malnutrition or Loeffler's pneumonia (fever, cough and eosinophilia due to a heavy larvae burden). There are, however, reports of ascariasis in the biliary and pancreatic ducts that cause complications.(1-6).

\section{Case Report}

A 34-year-old woman was hospitalized for recurrent acute pancreatitis with complaint of severed upper abdominal pain. Vital sign on admission was stable. Per abdomen examination revealed tender over the epigastric region. Serum amylase on admission is $1684 \mathrm{U} / \mathrm{L}$. She had previous admissions in the past two weeks ago with similar complaints. She had been worked up extensively for abdominal pain and was diagnosed as having idiopathic pancreatitis. She had no other medical history of note and did not consume alcohol. Patient was scheduled for urgent ultrasound hepatobiliary system . The finding was revealed dilated intrahepatic and common bile duct with tubular structure with segmentation noted within the common bile duct. Movement noted in keeping with viable biliary ascariasis. (Figure 1).Endoscopic Retrograde Cholangio Pancreatography ( ERCP ) was plan for the patient next day. Two Ascaris worms was completely removed during the procedure ( Figure 2 ). Cholangiogram post worm removal showed complete clearance .Antihelminthic medicine was prescribed for the patient and the worm histology confirmed it to be Ascaris lumbricoides. The patient subsequently recovered and she was well when discharged.. The patient was followed up for one year, and as she remained asymptomatic with no recurring attacks of abdominal pain, she was discharged from further follow-up.

\section{Discussion}

Ascaris lumbricoidesis a common parasitic infestation that is known to infect more than a billion people worldwide.(3) Endemic areas include tropical and subtropical countries. The warm and humid climate is suitable for the growth and maturity of the larva. This parasite is transmitted through the faecal oral route and commonly infects populations with poor sanitation and hygiene. The egg hatches in the duodenum, and the larva penetrates the mucosa to enter the blood stream. The larva reaches the lung alveolus and migrates to the oropharynx. Then it is swallowed and matures into an adult worm in the gastrointestinal system again to feed off the host's ingested food.

Common clinical manifestations of this parasite include malnutrition, symptoms of intestinal obstruction and also pneumonitis, if the larvae load is high. However, there are also many case reports of this parasite obstructing the biliary tree, causing cholangitis and pancreatitis. Ultrasonography of the biliary system is the investigation of choice to reveal the aetiology of pancreatitis. It is capable of detecting stones and has been shown to be able to detect ascariasis.(4) ERCP acts as a good follow-up procedure that is both therapeutic and diagnostic. It is able to find the cause of pancreatitis in $50 \%$ of those that remain subtle with an ultrasonography. Most intraluminal obstructions are removable with the ERCP. There is a postulated female preponderance for biliary ascariasis due to the ampullary smooth muscle relaxing effect of the hormone progesterone.

Our patient presented with sudden episodes of pancreatitis, for which the aetiology was initially unknown. Sandouk et al reviewed 300 patients with pancreatic ascariasis in Syria and showed that ultrasonography, together with clinical findings, are the mainstay of diagnosing pancreatic ascariasis.(4). The studies mentioned above reported that ERCP is the standard treatment for biliary ascariasis. In cases where the worm cannot be reached by conventional ERCP, Sandouk et al have suggested using the whirlpool jet technique.(7) Following the extraction of the worm and the subsequent administration of anti-parasite medicine, the patient went on to be well at discharge. 

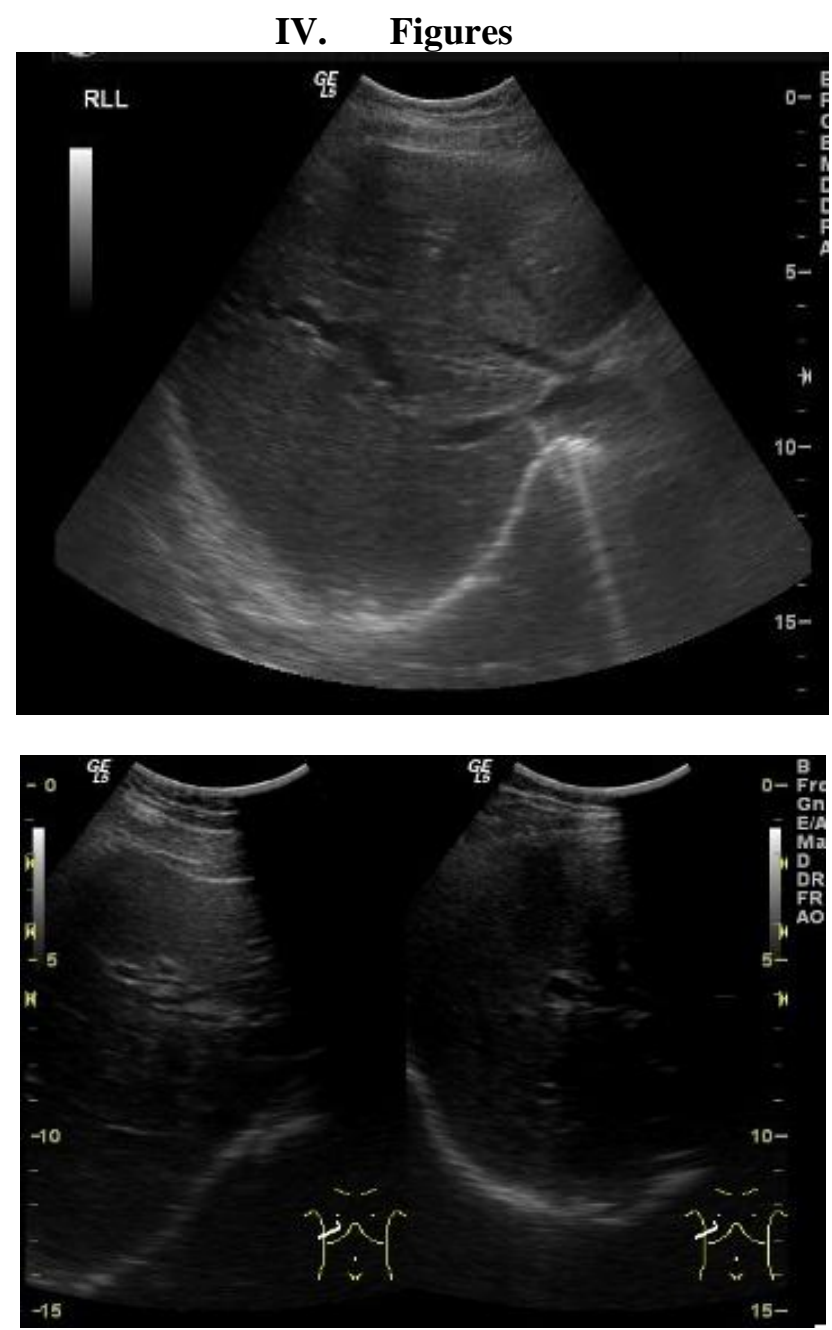

Figure 1 and 2 : ultrasound of abdomen showed dilated part of intrahepatic duct

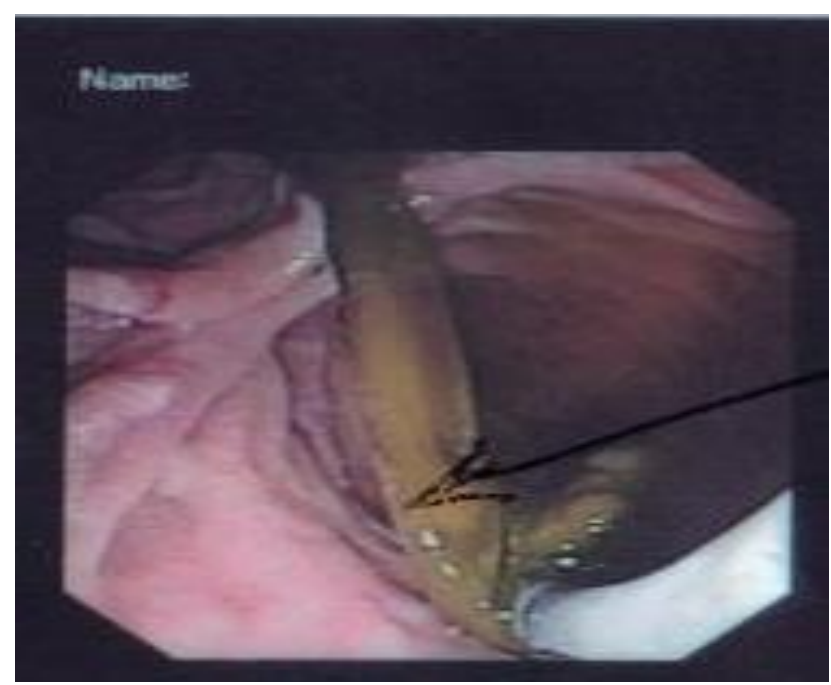




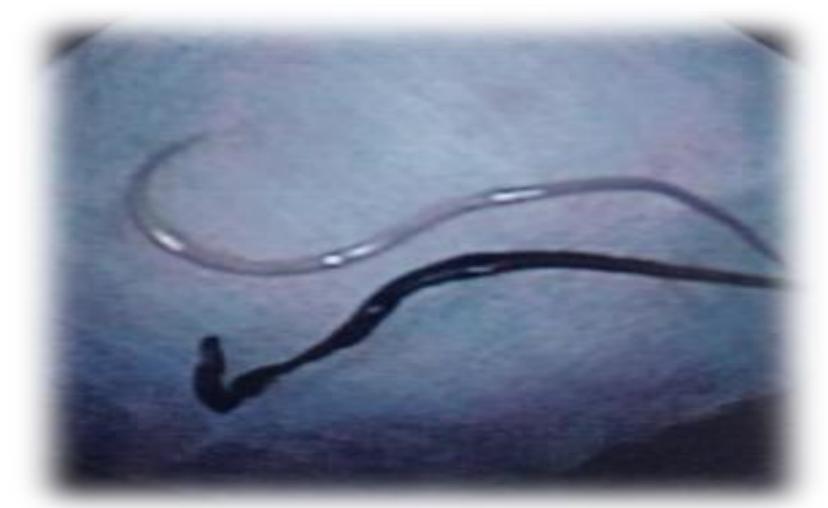

Figure3 and 4 :Ascariasis completely removed via ERCP

\section{References}

[1]. Misra SP, Dwivedi M. Clinical features and management of biliary ascariasis in a non-endemic area. Postgrad Med J2000; 76:2932.

[2]. Agaoglu N, Arslan MK. Recurrent attacks of acute pancreatitis from Ascaris in the common bile duct. J Am CollSurg2006; 203:580-1.

[3]. Clinch CR, Stephens MB. Case description of Ascariasis. Arch Fam Med2000; 9:1193-4.

[4]. Sandouk F, Haffar S, Zada M, Graham DY, Anand BS. Pancreatic-biliary ascariasis: experience of 300 cases. Am J Gastroenterol1997; 92:2264-7.

[5]. Khan BA, Shadan A, Suhail M, et al. Magnetic resonance cholangiography in biliary ascariasis. JK Pract2005; 12:205-6.

[6]. Kumar VH, Kamala CS. Biliary ascariasis. Indian Pediatr 2004; 41:1273-4.

[7]. Sandouk F, Anand BS, Graham DY. The whirlpool jet technique for removal of pancreatic duct ascaris.GastrointestEndosc1997; 46:180-2. 